\title{
Phosphor-Free GaN-Based Transverse Junction Light Emitting Diodes for the Generation of White Light
}

\author{
J.-W. Shi, H.-Y. Huang, C.-K. Wang, J.-K. Sheu, W.-C. Lai, Y.-S. Wu, C.-H. Chen, J.-T. Chu, H.-C. Kuo, \\ Wei-Ping Lin, Tsung-Hsun Yang, and J.-I. Chyi
}

\begin{abstract}
We demonstrate a GaN-based phosphor-free near-white-light light-emitting-diode (LED) structure that operates in the visible wavelengths and offers broadening and flattening optical bandwidth performance. The incorporation of GaN-based dual wavelengths (blue and green) multiple-quantum-wells with a transverse p-n junction produces a device which can directly generate stable and near visible white-light emissions. The shape of the optical spectra $(440-560 \mathrm{~nm})$ are invariable from low to very high levels of bias currents. The problems of nonuniform carrier distribution and bias dependent electroluminescence spectra that occur in traditional phosphor-free white-light or near-white-light LEDs (with vertical p-n junctions) are eliminated by the demonstrated structure.
\end{abstract}

Index Terms-GaN light-emitting-diode (LED), white-light generation.

\section{INTRODUCTION}

$\mathbf{M}$ $\mathrm{UCH}$ attention has been given and a huge impact on the solid-state lighting market has been made by GaN-based light-emitting-diodes (LEDs) that function in the visible or ultraviolet (UV) wavelengths. Commercial white-light LEDs are usually composed of GaN LEDs, which function in the blue or UV wavelengths [1], [2], and a mixture of red and yellow phosphor. However, due to loss during the processes of optical pumping and re-emission, the internal quantum efficiency of this kind of phosphor-converted white-light LED is sacrificed. In addition, the phosphor in discarded LEDs may lead to serious environmental protection problems. By utilizing the GaN-based multiple-quantum-wells (MQWs) with dual center wavelengths [3]-[5], Si and $\mathrm{Zn}$ codoped [6], [7] in MQWs, or cascade of green and blue GaN-based LEDs [8], white-light or near-white-light generation without using phosphor wavelength converter have been demonstrated. However, their output electroluminescence (EL) spectra are seriously dependent on the bias current [3]-[8]. Only white-light luminescence is exhibited

Manuscript received August 17, 2006; revised October 17, 2006. This work was supported in part by the National Science Council of Taiwan under Grant NSC-95-2215-E-008-008.

J.-W. Shi, H.-Y. Huang, C.-K. Wang, Y.-S. Wu, C.-H. Chen, and J.-I. Chyi are with the Department of Electrical Engineering, National Central University, Tainan 701, Taiwan, R.O.C. (e-mail: jwshi@ee.ncu.edu.tw; chyi@ee.ncu.edu. tw).

J.-K. Sheu and W.-C. Lai are with the Institute of Electro-Optical Science and Engineering, National Cheng-Kung University, Tainan 701, Taiwan, R.O.C. (e-mail: jksheu@mail.ncku.edu.tw).

J.-T. Chu and H.-C. Kuo are with the Department of Photonics, National Chiao-Tung University, Hsinchu 300, Taiwan, R.O.C.

W.-P. Lin and T.-H. Yang are wih the Department of Optics and Photonics, National Central University, Taoyuan 320, Taiwan, R.O.C.

Color versions of Figs. 1 and 2 are available at http://ieeexplore.ieee.org.

Digital Object Identifier 10.1109/LPT.2006.887362

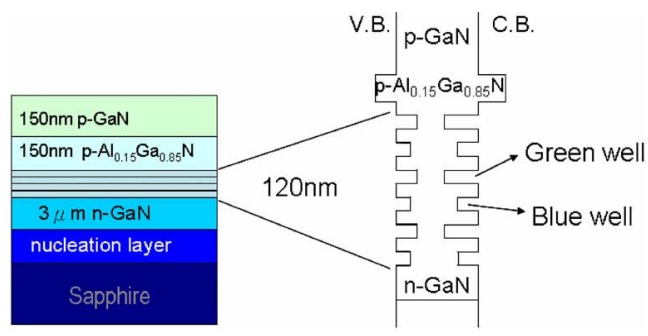

Fig. 1. The conceptual band diagram and the adopted epi-layer structure of the demonstrated near-white-light LED. C.B. and V.B. indicate the conduction band and valence band, respectively.

under low bias current due to the nonuniform distribution of injected carriers in the MQW layers [3]-[8] (or the saturation of the donor-to-acceptor transitions [6], [7] in the case of Si and $\mathrm{Zn}$ co-doped LEDs). In this paper, we discuss a novel structure for a phosphor-free white-light LED. Transverse $p-n$ junctions are incorporated into $\mathrm{GaN}$-based dual wavelength (blue and green) MQWs. The optical spectra $(440 \mathrm{~nm}-560 \mathrm{~nm})$ of the near-white-light emitted by the device are almost invariable, from low to high levels of bias current. The bias independent performance of the optical spectra of the demonstrated device can be attributed to the uniform distribution of the injected carriers in each quantum-well $(\mathrm{QW})$ with different center wavelengths.

\section{DEVICE STRUCTURE AND FABRICATION}

Fig. 1 shows the conceptual band diagram and the adopted epi-layer structure of the demonstrated device. The epi-layer structures are grown by metal-organic-chemical-vapor-deposition (MOCVD) on a sapphire substrate. The active MQW layers are composed of interlaced blue $(\sim 450 \mathrm{~nm})$ and green $(\sim 560 \mathrm{~nm}) \quad \mathrm{In}_{x} \mathrm{Ga}_{1-x} \mathrm{~N}-\mathrm{GaN}$-based QWs, sandwiched between upper p-type $\mathrm{GaN}$ and lower n-type GaN layers [3], [4]. The numbers of blue and green wells are the same, i.e., equal to three. Fig. 2(a) and (b) shows the top-view and conceptual cross-sectional view of the fabricated device. As shown in Fig. 2(a), the top p-metal $(\mathrm{Ni}-\mathrm{Au})$ circle has a diameter of $150 \mu \mathrm{m}$ and is surrounded by square n-metal contacts on the same plane. The planar p-n junctions were created by the Si-implantation technique. The proper implant dose/energy was adopted and thermal annealing at around $1000{ }^{\circ} \mathrm{C}$ was used to invert the desired topmost p-type GaN layer to an n-type [9]. After the implantation process, a ring-shape was trenched into the upper p-type GaN layer, as shown in Fig. 2(a). This acted to force the injected current through the buried MQWs after which we did a metallization process on the $\mathrm{p}$ - and $\mathrm{n}$ - contact layers. The total active area of our device, as shown in Fig. 2, 


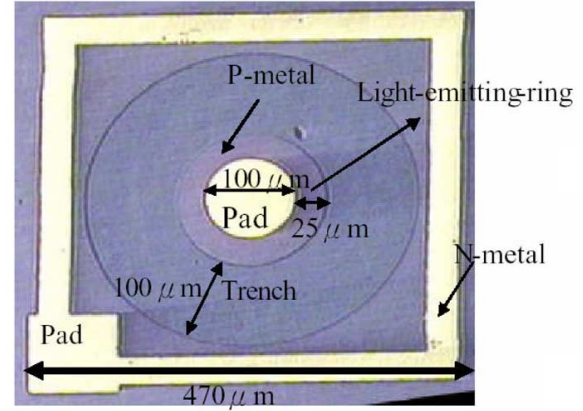

(a)

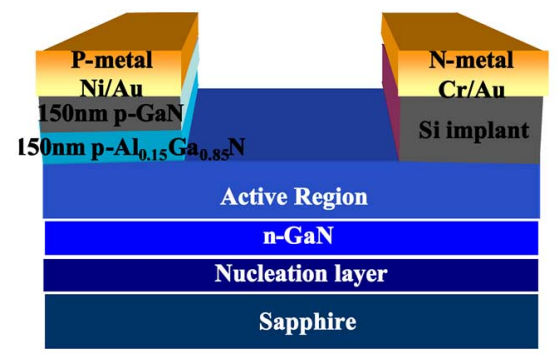

(b)

Fig. 2. The top-view (a) and conceptual cross-sectional view (b) of the demonstrated near-white-light LED. The trench-widths of devices A, B, and C are 100, 50 , and $25 \mu \mathrm{m}$, respectively. The light-emitting area of devices A, B, and C are 9800,41200 , and 62800 , respectively.

is around $470 \mu \mathrm{m} \times 470 \mu \mathrm{m}$. In order to perform the on-wafer probing and measurement of the fabricated device, a circular n-type metal contact, with an Au thickness of $\sim 300 \mathrm{~nm}$ and $100 \mu \mathrm{m}$ in diameter was deposited on the thin $\mathrm{Ni}-\mathrm{Au}$ p-metal circle. Details of the geometry and size of the fabricated device are shown in Fig. 2(a). The overall fabrication-processes are similar to those of the transverse-junction-stripe lasers [10], [11], which have a transverse $p-n$ junction to inject the bias current in the lateral direction. Under a forward bias, strong near-white-light emission can be observed between the two circles in the $\mathrm{Ni}-\mathrm{Au}$ p-metal region and the p-pad; there is no output light from the trench region making the total effective light-emitting region ring-shaped. The output power and EL spectral performance of devices fabricated with different trench-widths and effective light-emitting areas are studied. The trench-widths of devices A, B, and C are 100, 50, and $25 \mu \mathrm{m}$, respectively, and their corresponding light-emitting areas are given in the captions of Fig. 2. The inset in Fig. 3 shows the measured current-voltage $(I-V)$ curves of these three devices. All three devices show rectifying behaviors with a turn-on voltage of around $4 \mathrm{~V}$. The measured differential resistance of device $\mathrm{C}$, with a $4-\mathrm{V}$ turn-on voltage, is around $98 \Omega$. We achieved a similar I-V performance to that achieved in previous work on GaN planar p-n junctions and such performance may be improved by passivating the p-type GaN surface during thermal annealing processes [9]. Our device has a much larger turn-on voltage ( $4 \mathrm{~V}$ versus $3 \mathrm{~V}$ ) and differential resistance (98 $\Omega$ versus $12 \Omega$ ) compared to the I-V performance of the state-of-the-art phosphor-using vertical white-light LEDs with a very similar active area $(300 \mu \mathrm{m} \times 400 \mu \mathrm{m})$. Furthermore, our ideality factor value $(\sim 5)$ is around two times larger than

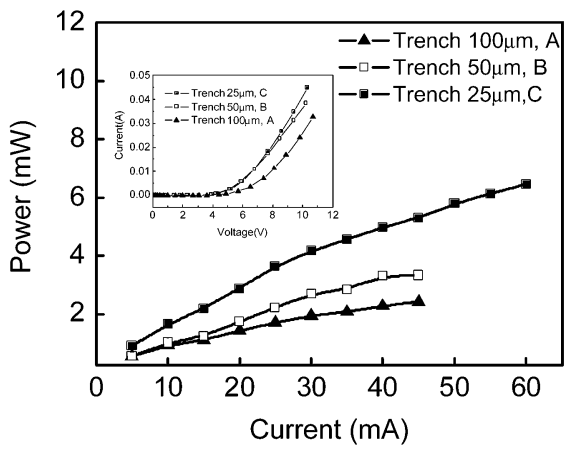

Fig. 3. The measured output power versus bias current of devices A, B, and C at room temperature. The propagation loss $(3 \mathrm{~dB})$ of the optical fibers has been corrected. The inset shows the measured $I$ - $V$ curves of these three devices.

that of (2.5) reported for phosphors-free vertical GaN LEDs fabricated with the same epi-layer structure as our device [4]. This phenomena may originate from damage done to the p-type GaN layer during the thermal annealing process resulting in degradation of the p-type Ohmic contacts. On the other hand, our device exhibits a smaller leakage current density, under the same reverse bias voltage $(-1 \mathrm{~V}),\left(5.84 \mathrm{nA} / \mathrm{cm}^{2}\right.$ versus $\left.18.75 \mathrm{nA} / \mathrm{cm}^{2}\right)$ than does the phosphors vertical white-light LEDs, which may be attributed to the fact that our device has a planar rather than an etched-mesa structure, meaning that the leakage current from the etched sidewall can thus be neglected. The major difference between the traditional LEDs or super-luminescence-diodes and our demonstrated device, is that in our structure the $p-n$ junction is located in the transverse direction, and that the lateral injection current will result in a uniform distribution of injected carriers in each QW layer with different center wavelengths [12]. The problems of nonuniform gain spectrum and carrier distribution encountered in the traditional devices, which can be attributed to the accumulation of injected carriers near the p-side QWs [3]-[8], [13], can thus be eliminated.

\section{MEASUREMENT RESUlts AND DisCUSSION}

Fig. 3 shows the measured output power versus bias current of devices A, B, and C. The maximum bias current of each device is limited by its failure. The output optical power was collected using a multi-mode optical fiber, which was connected with an optical spectrum analyzer (ANDO AQ-6315A) to record the power and spectra directly. During measurement, the devices were simply placed on a metal chunk without any cooling system. It can clearly be seen that the output power and external efficiency of device $\mathrm{C}$ is the highest, due to its having the largest emitting area and smallest trench-width among the three devices. The total collected optical power of device $\mathrm{C}$ is around $6.5 \mathrm{~mW}$ (under a 60-mA bias current) after correcting for an approximately 3 -dB propagation loss of the optical fiber. Compared with the output power performance of the state-of-the-art vertical phosphor white-light LEDs in a high-power package, device $\mathrm{C}$ exhibited around half $(\sim 0.45)$ of the output power density (output power/light-emitting active area), given the same measurement setup and bias current. Moreover, the power performance of our primarily demonstrated device can be improved by utilization of the proper device package, reducing the contact resistance, and minimizing the heating effect. All three devices 

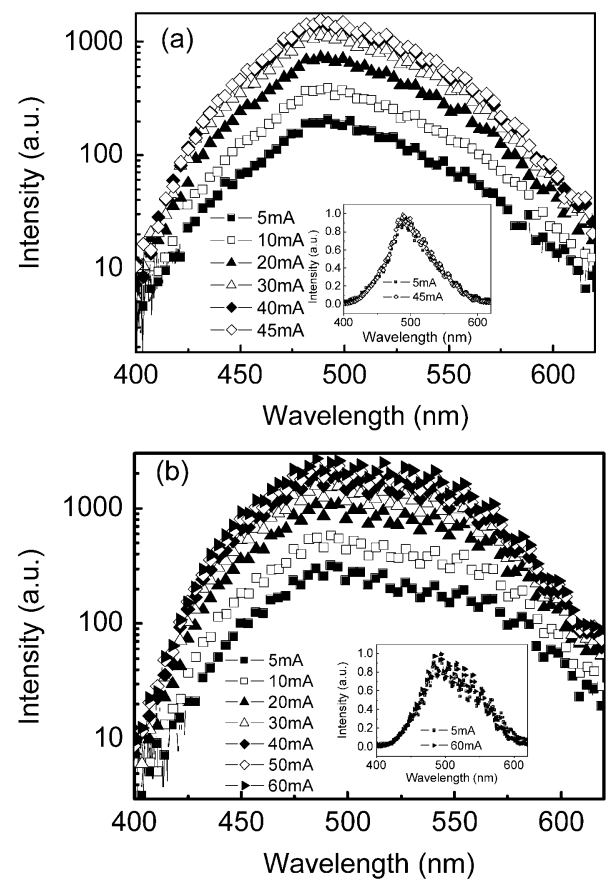

Fig. 4. The measured EL spectra of (a) device B and (b) device $\mathrm{C}$ (b) under different bias currents. The insets to (a) and (b) show the same spectra after normalization.

perform similarly in terms of the output EL spectra, except for a slight difference in the center wavelengths. Fig. 4(a) and (b) shows the measured EL spectra of devices B and C for different bias currents. The slight difference in the center wavelengths may be attributed to the nonuniformity of the MOCVD-grown epi-layers. The insets in Fig. 4 show the same spectra after normalization; each trace is normalized to its maximum. In Figs. 4(a) and (b) and their inset, we can clearly see that the shape of the measured spectra of both devices does not change with the bias current and exhibits only a broad peak with a wide optical bandwidth (440-560 nm), even under highest bias current (60 mA for device C). We can, thus, conclude that with a relatively narrow trench-width (25 $\mu \mathrm{m}$ versus $100 \mu \mathrm{m})$, not only can we sustain a wide optical bandwidth, but the output optical power is also improved. The performance of the EL spectra achieved is superior to that reported for phosphor-free whitelight GaN LEDs [3]-[8], which usually exhibit much more serious bias dependent EL spectra, or the blue or green-emission lines dominate the measured spectra [3]-[8], due to the accumulation of injected holes near the p-side QWs [3]-[8], [13] under high bias current. According to the measured bias-insensitive EL spectra and CIE chromaticity diagram, the calculated color coordinates of device $\mathrm{C}$ will be close to that of the white-light region of the CIE diagram, varying slightly from $x=0.23$ and $y=0.42$ to $x=0.2$ and $y=0.42$ when the bias current increases from $5 \mathrm{~mA}$ to $60 \mathrm{~mA}$. In order to further achieve visible white-light emission, the numbers of wells with different center wavelengths must be optimized. The uniform distribution of injected carriers in the active region of our demonstrated trans- verse junction LED allows us to avoid the problems of parasitic QW layers [13] and to achieve the desired color temperature and color coordinates.

\section{CONCLUSION}

In conclusion, by combining dual-wavelength GaN-based MQWs with the transverse p-n junction; we demonstrate a device that can generate stable and invariable near-whit-light spectra under a wide range of bias currents, without using a phosphor wavelength converter. This excellent bias insensitive performance of the EL spectra can be attributed to the transverse p-n junctions, which allow for a uniform distribution of injected carriers in each well layer with different center wavelengths.

\section{REFERENCES}

[1] S. J. Chang, W. C. Lai, Y. K. Su, J. F. Chen, C. H. Liu, and U. H. Liaw, "InGaN/GaN multiquantum well blue and green light emitting diodes," IEEE J. Sel. Topics Quantum Electron., vol. 8, pp. 278-283, Mar./Apr. 2002.

[2] J. K. Sheu, S. J. Chang, C. H. Kuo, Y. K. Su, L. W. Wu, Y. C. Lin, W. C. Lai, J. M. Tsai, G. C. Chi, and R. K. Wu, "White-light emission from near UV InGaN-GaN LED chip precoated with blue/green/red phosphors," IEEE Photon. Technol. Lett., vol. 15, no. 1, pp. 18-20, Jan. 2003.

[3] Y.-L. Li, Th. Gessmann, E. F. Schubert, and J. K. Sheu, "Carrier dynamics in nitride-based light-emitting p-n junction diodes with two active regions emitting at different wavelengths," J. Appl. Phys., vol. 94 , pp. 2167-2172, Aug. 2003

[4] S. C. Shei, J. K. Sheu, C. M. Tsai, W. C. Lai, M. L. Lee, and C. H. Kuo, "Emission mechanism of mixed-color $\mathrm{InGaN} / \mathrm{GaN}$ multi-quantum-well light-emitting diodes," Jpn. J. Appl. Phys., vol. 45, no. 4A, pp. 2463-2466, Apr. 2006.

[5] H.-S. Chen, D.-M. Yeh, C.-F. Lu, C.-F. Huang, W.-Y. Shiao, J.-J. Huang, C. C. Yang, I.-S. Liu, and W.-F. Su, "White light generation with CdSe-ZnS nanocrystals coated on an InGaN-GaN quantum-well blue/green two-wavelength light-emitting diode," IEEE Photon. Technol. Lett., vol. 18, no. 13, pp. 1430-1432, Jul. 1, 2006.

[6] S. J. Chang, L. W. Wu, Y. K. Su, C. H. Kuo, W. C. Lai, Y. P. Hsu, J. K. Sheu, J. F. Chen, and J. M. Tsai, "Si and Zn co-doped InGaN-GaN white light-emitting diodes," IEEE Trans. Electron Devices, vol. 50, no. 2, pp. 519-521, Feb. 2003.

[7] J. K. Sheu, C. J. Pan, G. C. Chi, C. H. Kuo, L. W. Wu, C. H. Chen, S. J. Chang, and Y. K. Su, "White-light emission from InGaN/GaN multi-quantum well light-emitting diodes with $\mathrm{Si}$ and $\mathrm{Zn}$ codoped active layer," IEEE Photon. Technol. Lett., vol. 14, no. 4, pp. 450-452, Apr. 2002.

[8] C. H. Chen, S. J. Chang, Y. K. Su, J. K. Sheu, J. F. Chen, C. H. Kuo, and Y. C. Lin, "Nitride-based cascade near white light-emitting diodes," IEEE Photon. Technol. Lett., vol. 14, no. 7, pp. 908-910, Jul. 2002.

[9] J. K. Sheu, C. J. Tun, M. S. Tsai, C. C. Lee, G. C. Chi, S. J. Chang, and Y. K. Su, " $\mathrm{n}^{+}-\mathrm{GaN}$ formed by Si implantation into $\mathrm{p}-\mathrm{GaN}$," J. Appl. Phys., vol. 91, pp. 1845-1848, Feb. 2002.

[10] Y. J. Yang, Y. C. Lo, G. S. Lee, K. Y. Hsieh, and R. M. Kolbas, “Transverse junction stripe laser with a lateral heterobarrier by diffusion enhanced alloy disordering," Appl. Phys. Lett., vol. 49, pp. 835-837, Oct. 1986.

[11] F. Brillouet, E. V. K. Rao, and J. Beerens, "New low capacitance transverse junction stripe $\mathrm{AlGaAs} / \mathrm{GaAS}$ laser for planar laser-MESFET integration," Electron. Lett., vol. 24, pp. 97-99, Jan. 1988.

[12] J.-W. Shi, T.-J. Hung, Y.-Y. Chen, Y.-S. Wu, W. Lin, and Y.-J. Yang, "InP-based transverse junction light-emitting-diodes for white-light generation at infrared wavelengths," IEEE Photon. Technol. Lett., vol. 18, no. 19, pp. 2053-2055, Oct. 1, 2006.

[13] C.-F. Lin, Y.-S. Su, C.-H. Wu, and G. S. Shmavonyan, "Influence of separate confinement heterostructure on emission bandwidth of InGaAsP superluminescent diodes/semiconductor optical amplifiers with nonidentical multiple quantum wells," IEEE Photon. Technol. Lett., vol. 16, no. 6, pp. 1441-1443, Jun. 2004. 